\title{
Retroperitoneal abscesses: Origin, ethiology and
}

\section{percutaneous treatment}

\author{
Rahmi Aslan ${ }^{1 *}$, Mesut Özgökçe ${ }^{2}$, Recep Eryılmaz ${ }^{1}$, Mehmet Arif Duran ${ }^{1}$ Murat Yıldızhan ${ }^{3}$ Kerem $^{1}$ \\ Taken $^{1}$
}

${ }^{1}$ Van Yuzuncu Yil University, Medical Faculty, Department of Urology, Van, Turkey

${ }^{2} V$ an Yuzuncu Yil University, Medical Faculty, Department of Radiology Van, Turkey

${ }^{3}$ Viransehir Medical Center, Department of radiology Sanliurfa, Turkey

\begin{abstract}
Retroperitoneal abscess is a rare clinical condition that can cause mortality if not properly treated. In our study, we aimed to report the data of retroperitoneal abscess cases which performed percutaneous drainage in the first step treatment.

A retrospective study was made of 81 patients with retroperitoneal abscesses treated at our hospital from June 2010 to December 2017 for the purpose of analyzing the diagnosis and treatment of these rare infections. In each case, we analyzed patient characteristics, abscess location and origin, predisposing factors, clinical presentation, microbiology, radiographic findings, treatment, and outcome.

81 cases were identified as $11(14 \%)$ primary and $70(86 \%)$ as secondary. The majority (63\%) of these abscesses were originated from Urinary System, while others were found to be originated from psoas, vertebral and Gastrointestinal system (GIS). Urolithiasis, previous surgery, and other Urinary system anomalies were determined to be the most common predisposing factors. Escherichia coli (E. coli) was the most commonly seen pathogen in abscess culture. In all patients, percutaneous drainage catheter was successfully placed in the abscess lodge. No serious complications occurred in patients during and after the procedure. 78/81 (96\%) clinical success was achieved. In 12 months follow-ups, recurrence was seen in only 5 patients.

Percutaneous abscess drainage should be preferred in the treatment of retroperitoneal abscesses as easy to perform, cost effective, low complication risk and high success rate
\end{abstract}

Key Words: Retroperitoneal abscesses, Percutaneous drainage, Origin, Ethiology

\section{Introduction}

Retroperitoneal abscesses (RPA) are rare but with high mortality and morbidity when not treated appropriately. They are classified as primary and secondary. It has been reported that when the primary abscesses occur hematogenously in the retroperitoneal (RP) location of microorganisms, the secondary abscesses develop due to organ pathologies associated with RP $(1,2,3)$.

Diabetes mellitus (DM), urinary obstructions, stone diseases, previous urological interventions and cirrhosis are the most common predisposing factors $(2,4-7)$.

Conventional abscess treatment is surgical drainage in the presence of antibiotics $(8,9)$. This method is known to increase morbidity and mortality $(10,11)$. In recent years, early diagnosis and percutaneous drainage have been provided, especially with the development of radiological imaging methods such as Ultrasound (US) and Computerized Tomography (CT). US or CTguided percutaneous drainage is currently recommended as an alternative to surgery with minimal invasive and high success rates $(12,13)$.

In this study, we aimed to share the data and experiences of our percutaneous treatment with the literature. Our work also has the widest range of series of published work.

\section{Material and Method}

We retrospectively evaluated the data of 91 cases with 51 men (mean age 55.48) and 40 women (mean age 48.17) who were diagnosed with Retroperitoneal abscess between June 2010 and December 2017. The ethical approval of this study is received from our own institution (25.04.2018; 003). Demographic data, location and origin of the abscess, predisposing factors, urine, blood and 
Table 1. Patients' characteristics

\begin{tabular}{ll}
\hline Variables & Total $\mathrm{n}=81$ \\
\hline Age (average) Gender & $53.4 \pm 17.3$ \\
Male (\%) & $46(57)$ \\
Female (\%) & $35(43)$ \\
Origin of the abscesses & \\
Urinary system (\%) & $51(63)$ \\
Renal (\%) & $35(69)$ \\
Perirenal(\%) & $16(31)$ \\
GIS (\%) & $3(4)$ \\
Psoas (\%) & $15(18,5)$ \\
Vertebral (\%) & $6(7)$ \\
Other (\%) & $6(7)$ \\
Symptoms & \\
Waist and side pain (\%) & $60(74)$ \\
Fever (\%) & $40(49)$ \\
Predisposing factors & \\
Uroloitiasis (\%) & $24(30)$ \\
Previous surgery (\%) & $23(28)$ \\
Urological & $16(70)$ \\
GIS & $3(13)$ \\
Vertebral & $4(17)$ \\
Urinary system anomalies $(\%)$ & $18(22)$ \\
Dm (\%) & $18(22)$ \\
\hline
\end{tabular}

abscess culture, microbiological agents, physical examination and radiological imaging findings, treatment methods and long-term follow-up results of all patients were examined (Table 1). 81 patients who underwent percutaneous drainage (who were determined to have abscess clinically and radiologically) were included in the study. The other 10 patients who were treated with antibiotics only, (abscess smaller than $3 \mathrm{~cm}$ ) and underwent surgical resection were excluded from the study.

Percutaneous drainage was performed using a 6 $\mathrm{MHz}$ convex probe US device in 71 patients (Siemens ACUSON S2000 TM, Siemens Healthcare, Erlangen, Germany). The other 10 abscesses, deeply located and not sonographically clear, Percutaneous drainage was performed in 16section CT (Siemens Medical system, Germany). In all procedures 10-12-14 F catheter (angiotech) was used as Drainage catheter according to the density of abscess. Percutaneous abscess drainage was performed under local anesthesia using the Seldinger method under US or CT guidance. In order not to damage the vascular and adjacent organs, the shortest distance between the abscess and the skin was preferred, and the location of the
Table 2. Organisms causing RPA abscesses

\begin{tabular}{lc}
\hline Microorganisms & $\begin{array}{c}\text { Number of patients } \\
\mathrm{n}=55(\%)\end{array}$ \\
\hline Escherichia coli & $27(49)$ \\
Klebsiella pneumonia & $8(15)$ \\
Pseudomonas & $6(11)$ \\
aeruginosa & \\
Staphylococcus aureus & $5(9)$ \\
Polymicrobial & $4(7)$ \\
Mycobacterium & $3(5)$ \\
Proteus mirabillis & $2(4)$ \\
\hline
\end{tabular}

entry to the abscess was determined. After the necessary sterilization and preparation, approximately $10 \mathrm{cc}$ of anesthetic (prilocaine hydrochloride) was delivered subcutaneously and muscle tissues, then entered the abscess pouch with an access needle. After the guide wire has been delivered and the dilatation has been attained with the dilator the drainage catheter has been placed on the abscess pouch over the pre-existing guide wire. All catheters were fixed with $3 / 0$ silk on the skin.

The sample was taken for microbiological evaluation after the drainage catheter was attached. Drainage catheters were washed daily with saline and the abscess cavity was checked on days 3 and 7 with US. The catheter was withdrawn when the total daily drainage fell below $10 \mathrm{cc}$.

\section{Results}

81 patients with a RPA of over $3 \mathrm{~cm}$ were included in the study. All patients received empirical treatment with broad spectrum antibiotics. Then the patient was treated according to the antibiogram of the fluid collected during the drainage procedure. A total of 81 cases were identified, $11(14 \%)$ as primary and $70(86 \%)$ as secondary. 51 of these abscesses (63\%) were localized from urinary system (35 perirenal, 16 renal) and $15(19 \%)$ patients had abscess in psoas muscle. Five of these patients had primary abscess and the other 10 patients had secondary abscess. In $6(7 \%)$ patients, a vertebral-originated abscess was detected in the posterior pararenal area. These included vertebral surgery in 2 patients and vertebral trauma in 1 patient. There were $3(4 \%)$ abscesses suspected to be of GIS origin and 2 of them had history of hepatobiliary surgery and a colonic surgery. Origin of 6 abscesses (7\%) could not be determined (Table 1).

Predisposing factors were renal and ureteral 
Aslan et al / Percutaneous Drainage of Retroperitoneal Abscesses

Table 3. Interventions and Recurrence Rates after Percutaneous Drainage

\begin{tabular}{lccccc}
\hline & $\begin{array}{c}\text { Total } \\
\mathrm{n},(\%)\end{array}$ & $\begin{array}{c}\text { US } \\
\mathrm{n},(\%)\end{array}$ & $\begin{array}{c}\text { GIS } \\
\mathrm{n},(\%)\end{array}$ & $\begin{array}{c}\text { Musculoskeletal } \\
\mathrm{n},(\%)\end{array}$ & $\begin{array}{c}\text { Other } \\
\mathrm{n},(\%)\end{array}$ \\
\hline The number patients & 81 & $51(63)$ & $3(4)$ & $21(26)$ & $6(7)$ \\
Nephrectomy & $12(15)$ & $11(14)$ & $1(1)$ & & \\
PNL URS & $20(25)$ & $20(25)$ & & & \\
Hemicolectomia & $1(1)$ & & 1 & $2(2)$ & 0 \\
Surgical debridement & $5(6)$ & $3(4)$ & 0 & $2(2)$ & 0 \\
Recurrence & $5(6)$ & $3(4)$ & 0 & & \\
\hline
\end{tabular}

US: Urinary system GIS: gastrointestinal system PNL: Percutaneous nephrolithotomy URS: Ureterorenoscopy

calculus in $24(30 \%)$ patients and $23(28 \%)$ patients had previous surgical history. 18 (22\%) patients had other urinary system anomalies (Uretro-Pelvic junction (UPB) stenosis, VUR, ureteral obst ...) $18(22 \%)$ patients had DM and 11 $(14 \%)$ patients had no predisposing factors. No immunosuppressive causes such as chemotherapy radiotherapy and HIV were detected in any of our patients (Table 1).

In $60(74 \%)$ of the patients, side and back pain was the most common symptom, while $40(49 \%)$ of the patients had fever. Abdominal and pelvic pain, lower extremity numbness, weight loss, loss of appetite and fatigue were less common symptoms.

Escherichia coli (E. coli) was found in $27(\% 49)$ of the 55 patients whose abscess culture was positive. Staphylococcus aureus was seen in 5 $(\% 9)$ patients, Klebsiella pneumoniae in $8(15 \%)$ patients, Mycobacterium (TBC) in $3(\% 5)$ patients, Pseudomonas aeruginosa in $6(\% 11)$ patients and polymicrobial growth in $4(\% 7)$ patients (aerobic and anaerobic bacteria) and Proteus Mirabilis in 2 $(\% 4)$ patients. Abscess originated from urinary system was present in $18(\% 67)$ patients with E. Coli and in all patients with Klebsiella pneumoniae and Pseudomonas aeruginosa. Polymicrobial proliferation was observed in 3 patients with GIS, whereas Staphlococcus aureus ( $27 \%$ in 4 patients) was the most common pathogen in the psoas abscess. TBC was detected in 2 vertebral abscess and 1 psoas abscess (Table 2).

E. coli $(50 \%)$ was the most frequent pathogen in urine cultures and less frequently in Klebsiella pneumoniae, Pseudomonas aeruginosa and other pathogens.

In all patients, percutaneous drainage catheter was successfully placed in the abscess lodge. The removal time of the catheters after percutaneous drainage was calculated as mean $11.2 \pm 3.6$ days. The liquid from the drainage catheters was followed daily and the catheter was withdrawn when the 24-hour flow rate dropped to $10 \mathrm{cc}$ or less. No serious complications developed during or after the procedure. Complete recovery of the clinical presentation, complete disappearance of the abscess, or shrinkage was considered a clinical success. $78 / 81 \quad(96 \%)$ clinical success was achieved. Three patients without clinical improvement were treated surgically

In first-line treatment, we administered percutaneous drainage with antibiotic therapy. In the elective condition, the second-line medical and surgical treatments (Table 3) were applied based on the basic reason. Long-term (9 months-1 year) anti-tuberculosis treatment was given to 3 patients who were diagnosed with tuberculosis. Treatment of DM-diagnosed patients was regulated. In 12 months-follow up 5 patients had demonstrated recurrence. These patients underwent surgical drainage

\section{Discussion}

Retroperitoneal abscesses are rare clinical conditions that cause high mortality and morbidity when not properly treated (3). In the past, it has been reported that primary retroperitoneal abscesses are frequently seen, and most of them are due to staphylococci originating from a foci on the skin (2). Due to the widespread use of antibiotic drugs, the incidence of primer abscesses is decreased, but nowadays, secondary abscesses due to gram-negative basilli are more frequent and they are frequently caused by genito uriner system (GUS) and GIS (2-4). In our study, $86 \%$ of our cases were secondary consistent to the literature. When we classified the cases according to the abscess origin, $63 \%$ of the patients were originated from GUS, $26 \%$ from musculoskeletal, $4 \%$ from GIS and $7 \%$ of them were not identified. Therefore, when retropeitoneal abscess is seen, secondary causes must be kept in mind

The majority of RP abscesses develop secondary to urinary tract infections, which are caused by 
gram-negative bacteria. Urinary system stone disease, Urinary system obstructions and previous urological surgeons are considered as the main risk factors (2-14,15).Shu et al. (16) reported that $52 \%$ of patients with retroperitoneal abscesses had urinary system origin. Four of these patients $(26 \%)$ had previous urological surgery and 2 patients $(13 \%)$ had urinary system stone disease. However, the number of cases in this study seems to be very small. In a study by Capitán Manjón et al. (2) named Retroperitoneal abscesses: Analysis of 66 cases, $72 \%$ of the RPAs were determined to be derived from the urinary system. Of these cases, urolithiasis was detected in 48\%, and urological surgery in $23 \%$ and these were reported to be the most important predisposing factors. In our study, 63\% of the abscesses were found to be originated from the urinary system. Urolithiasis was present in $30 \%$ of patients, urological surgeries in $20 \%$ patients and other urological anomalies in $22 \%$ patients. Urolithiasis was the most common cause of underlying disease. We think that RPA cases should be examined especially in terms of urinary system stone and other urinary system anomalies

The main treatment of retroperitoneal abscesses is percutaneous or surgical drainage. Undrained abscesses are $80 \%$ to $100 \%$ mortal. These rates are significantly reduced with surgical treatment (1720). However, in recent years, percutaneous drainage in the context of imaging has been implicated in first-line treatment of intracorporeal fluid collections with minimal invasive and low complication rates (20). Abscesses under $3 \mathrm{~cm}$ can only be treated with antibiotics, but in larger abscesses, surgery or percutaneous drainage is recommended (14). In this study, we performed percutaneous drainage as the primary treatment for all patients with abscesses of over $3 \mathrm{~cm}$. Clinical success was achieved in $96 \%$ of these patients $(78 / 81)$,

Urinary system-derived abscesses are classified as renal, pararenal and mixed type abscesses (15). The best clinical approach for these abscesses treatment is still being discussed. Only Antibiotherapy is recommended in abscesses smaller than $3 \mathrm{~cm}$, but percutaneous or surgical treatment should be performed because the antibiotic is insufficient in the larger abscesses (21). In our series, the urinary system-derived abscesses were identified as 31\% renal and 69\% as perirenal type. In a study by Coelho et al. [15] in $83 \%$ of patients who underwent percutaneous drainage in a urinary system-based abscess were cured while cure rates after surgical drainage were $58 \%$. In a similar study [4] published in 2008,
$35.9 \%$ of patients were treated with only antibiotics, $54 \%$ with percutaneous drainage, $7.2 \%$ with nephrectomy, and 3.6\% with surgical drainage. In this study, $96.3 \%$ clinical improvement was achieved after treatment and 3 patients $(5.4 \%)$ died due to sepsis. In our study, percutaneous drainage was performed as the firstline treatment in all patients with abscesses of over $3 \mathrm{~cm}$ of urinary system origin. In 50 of the 51 patients with renal perirenal abscesses (98\%) clinical improvement was achieved, whereas in only one patient, when contrast material was applied to the catheter and the abscesses of the patient was found to be fistulized to colon. This patient underwent hemicolectomy and nephrectomy as surgical treatment. After this first step treatment 11 patients underwent nephrectomy (non-functioning kidney) and 20 patients underwent stone surgery (PNL, URS) directed at the underlying cause in elective conditions. In our study, no serious complication such as sepsis or similar was seen in any patient after the first step of treatment with appropriate antibiotic and percutaneous drainage. After the interventions for the underlying cause with the second-line treatment, in the 12 -month follow-up only 3 patients developed recurrence. These patients were re-treated with surgical drainage

Retroperitoneal abscesses have been reported to be between $9 \%$ and $28 \%$ GIS source. A large proportion of this abscesses have been shown to develop secondary to necrotizing pancreatitis, appendicitis, and bowel perforation (2-16-21). In our study, only 3 (4\%) patients had GIS-induced abscesses, One of these patients which was secondary to colon surgery and the other two patients developed secondary to hepatocellular surgery.

Muscle skeletal abscesses are also frequently seen in Retroperitoneum. Psoas abscesses may be seen as primary or secondary to penetrating trauma, penetration of spinal osteomyelitis or tuberculosis, renal infection or inflammatory bowel disease $(22,23)$. Studies have indicated that antibiotic treatment with percutaneous drainage is safe and easy to perform in the treatment of psoas pyogenic abscesses $(23,24) .15$ of our patients had a psoas abscess. It was seen that 5 of them were primary, 9 of them were due to vertebral surgery, hip infection, trauma and one of them was due to tuberculosis spread. Percutaneous drainage was successfully applied to these abscesses. In followups recurrence was seen in 2 patients and they were treated with surgical drainage. 
The microorganisms seen in the retroperitoneal abscess can vary according to the origin of the abscess. Gram negative basils such as E. Coli, Klepsiella Pneumonia and Proteus Mirabilis are the most common pathogens in urinary-system originated abscesses. Aerobic and anaerobic polymicrobial proliferation is frequently seen in GIS-derived abscesses, whereas Mycobacterium tuberculosis and staphylococcus aurus are frequent pathologies in musculoskeletal abscess (2-16-22). In our series, E.Coli, Klebsiella Pneumonia, staphylococcus aurus and Polymicrobial bacteria were seen at similar rates in previous studies. Unlike previous studies, Pseudomonas was detected in $11 \%$ of patients, especially those undergoing urological calculus surgery. We think that this pathogen should be kept in mind when starting empirical therapy in such patients

The most common complication after retroperitoneal abscess treatment was abscess recurrence (12.4\%), whereas renal insufficiency pyelonephritis cutenous fistula was rarely reported (2). In our series, 5 (6.2\%) recurrences were seen during the 12 month follow-up after percutaneous drainage. three of these abscesses were originated from the urinary system whereas the other two were derived from psoas. We have not found any other complications in our follow-ups. We think that this depends on the appropriate treatment for etiology. To reduce recurrence and complications, drainage materials should be sampled and sent to culture

The most important limitations of our study are the retrospective nature of study and no comparison with the patients who underwent surgical drainage in the primary treatment.

In our study, which is one of the largest published series seen in RPA, Retroperitoneal abscess was the most common source of urinary system, whereas unlike previous studies, GIS derived abscesses were rarely detected. However, in this series, musculoskeletal abscesses have been found to constitute a significant part of the Retroperitoneal abscesses.

As a result, combined percutaneous drainage with antibiotics is an effective and reliable treatment, At the same time, evacuation of the infected material without spreading also reduces the risk of major complications such as sepsis and allows surgical treatment for underlying causes of abscesses to be performed in elective conditions.

\section{References}

1. Laganà $\mathrm{D}$, Carrafiello $\mathrm{G}$, Mangini $\mathrm{M}$, et al. Image-guided percutaneous treatment of abdominal-pelvic abscesses: a 5-year experience.[Article in English, Italian] Radiol Med 2008; 113: 999-1007.

2. Capitan Manjon C, Tejido Sanchez A, Piedra Lara JD, et al. Retroperitoneal abscesseseanalysis of a seriesof 66 cases. Scand J Urol Nephrol 2003; 37: 139-144.

3. Crepps JT, Welch JP, Orlando R. Management and outcome of Retroperitoneal abscesses. Ann Surg 1987; 205: 276-281.

4. Lee BE, Seol HY, Kim TK, et al. Recent clinical overview of renal and perirenal abscesses in 56 consecutivecases Korean J Intern Med 2008; 23: 140-148.

5. Fowler JE, Perkins T. Presentation, diagnosisand treatment of renal abscesses: 1972-1988. J Ural 1994; 151: 847-851.

6. Thorley JD, Jones SR, Sanford JP. Perinephric abscess. Medicine 1974; 53: 441-451.

7. Salvatierra O, Bucklew WB, Morrow JW. Perinephric abscess: a report of 71 cases. J Urol 1967; 98: 296-302.

8. Thanos L, Dailiana T, Papaioannou G, et al. Percutaneous CT-guided drainage of splenic abscess. AJR Am J Roentgenol 2002; 179: 629632.

9. Gerzof SG, Robbins AH, Birkett DH, Johnson WC, Pugatch RD, Vincent ME. Percutaneous catheter drainage of abdominal abscesses guided by ultrasound and computed tomography. AJR Am J Roentgenol 1979; 133: $1-8$.

10. Men S, Akhan O, Koroglu M. Percutaneousdrainage of abdominal abscess. Eur J Radiol 2002; 43: 204-218.

11. Cinat ME, Wilson SE, Din AM. Determinants for successful percutaneous image-guided drainage of intraabdominal abscess. Arch Surg 2002; 137: 845-849.

12. Edelstein H. Perinephric abscesses. Modern diagnosis and treatment in 47 cases. Medicine 1988; 67:118-131

13. Fowler $\mathrm{J}$ and Perkins T. Presentation, diagnosis and treatment of renal abscesses: 1972-1988. J Urol 1994; 151: 847-851.

14. Winter BM, Gajda M, Grimm MO Diagnosis and treatment of retroperitoneal abscesses.Urologe A 2016; 55: 741-747.

15. Coelho RF, Schneider-Monteiro ED, Mesquita JL, Mazzucchi E, Marmo Lucon A, Srougi M, Renal and perinephric abscesses: analysis of 65 consecutive cases World J Surg 2007; 31: 431436.

16. Shu HH, Wah OL, Chih ML et al. Retroperitoneal abscess: 7-year experience of 29 cases in a tertiary care center in Taiwan Urological Science 2015; 26: 218-221. 
17. Altemeier WA, Culbertsen WR, FullenWD, Shook CD. Intra-abdominalabscesses. Am J Surg 1973; 125: 70-79.

18. Fry DE, Garrison RN, Heitsch RC Calhoun K, Polk HC. Determinants of death in patients with intra-abdominal abscesses. Surgery 1980; 88: 517-523.

19. Gazelle GS, Mueller PR Abdominal abscesses. Imaging and intervention. Radiol Clin North Am 1994; 32: 913-932.

20. Hinsdale JG, Jaffe BM Reoperation for intraabdominal sepsis.Ann Surg 1984; 199: 3136.

21. Asai N, Ohkuni Y, Yamazaki I, Kaneko N, Aoshima M, Kawamura Y. Therapeutic impact of CT guided percutaneous catheter drainage in treatment of deep tissue abscesses.Braz J Infect Dis 2013; 17: 483-486.

22. Charalampopoulos A, Macheras A, Charalabopoulos A, Fotiadis C, Charalabopoulos K. Iliopsoas abscesses: diagnostic, aetiologic and therapeutic approach in five patients with a literature review.Scand J Gastroenterol 2009; 44: 594599.

23. Dinç H, Ahmetoğlu A, Baykal S, Sari A, Sayil O, Gümele HR. Image-guided percutaneous drainage of tuberculous iliopsoas and spondylodiskitic abscesses: Midterm results. Radiology 2002; 225: 353-358.

24. Yacoub WN, Sohn HJ, Chan S, et al. Psoas abscess rarely requires surgical intervention.Am J Surg 2008; 196: 223-227. 